\title{
Abandoning the blind legacy passed on horde of routine intra-abdominal drain insertion in cesarean section
}

\author{
Kamil M. Fram, Shawqi Saleh, Fidaa Thikerallah, Farah K. Fram, Rand K. Fram, Tamara Darwish, \\ Rana Haddad, Zeina Othman
}

Department of Obstetrics and Gynecology, Jordan University Hospital, Amman, Jordan

\begin{abstract}
Introduction: Cesarean section (CS) delivery is the most common major obstetrical surgical operation carried out in and is increasing in incidence throughout the world. The major involves some risks that might include: infection, coagulation problem, loss of blood, bowel or bladder injury, abnormalities of the placenta in subsequent pregnancies.

Aim of the study: To evaluate the clinical effectiveness of postoperative CS intra-abdominal drain insertion.

Material and methods: A prospective study was conducted on 245 patients in labor, at the Department of Obstetrics and Gynecology, Jordan University Hospital, between January 2017 and January 2018. Participants were divided into two groups: group I including those who had abdominal drains insertion during surgery and group II including women who had no abdominal drain inserted before closure. All patients on both groups were term pregnancies, underwent elective vs. emergency CS, and had no subcutaneous drains inserted.

Results: Clinical and surgical parameters were comparable in both groups. Postoperative hospital stay was significantly shorter in group II, whereas specific postoperative complication rate was significantly higher in group I. Drain site infection was noted in $2(1.6 \%)$, organ herniation in $2(1.6 \%)$, drain avulsion in $2(1.6 \%)$, severe pain at the site of insertion in $2(1.6 \%)$ patients.

Conclusions: Routine prophylactic intra-abdominal drain insertion post CS has no benefits and therefore should be stopped.
\end{abstract}

Key words: cesarean section, intra-abdominal drain, complications.

\section{Introduction}

Medicine is one of the most growing fields worldwide. A good surgeon must employ his time being updated, updating his knowledge and skills to keep up with this frighteningly growing trend, especially when it comes to the largest cavity in the human body: the abdomen.

Abdominal drainage is one of the oldest procedures in the art of surgery [1]. Controversy has been highlighting this procedure ever since its invention, and still continues. A hundred years ago, ardent enthusiasts for drainage, including Sir Lawson who dropped the famous saying: "When in doubt, drain!". However, huge figures in the field, including Sir Yates who introduced a new thought when he stated that: "Drainage of the general peritoneal cavity is a physical and physiological impossibility". Between these two spectrums lied many other opinions implying in the pros and cons of such technique including Joseph Price (1853-1911) whom quote: "There are those who ardently advocate it, there are those who in great part reject it, there are those who are lukewarm concerning it, and finally, some who, without convictions, are either for or against it as chance or whim, not logic may determine" [2]. A decades had passed on these great opinions, but no consensus has been made upon abdominal drainage up until the present moment; still surrounded by this great controversy and still an area of huge doubt.

The following article will try to lessen this unclarity and unravel some of the new evidence in this topic, with scarcity in research in this field; this work will set a new base from which other arguments may stem. This topic is of great importance, especially in which it's used in an urgent manner. It is worth highlighting that this topic is beyond the scope of this paper, especially that involves only cases of cesarean section (CS), albeit its importance as a starting point.

\section{Material and methods}

From January 2017 to January 2018, a total number of 2467 patients underwent CS at the Department of Obstetrics and Gynecology at Jordan University Hospi- 
tal. The study was carried out after the approval of the Institutional Review Board, the Ethics Committee and the Scientific Research Committee at our hospital. Data analysis was done using the Statistical Package for the Social Sciences (SPSS).

A total number of 245 patients were selected to be enrolled in this study with almost the same criteria of eligibility and exclusion, and all patients who needed subcutaneous drain insertion were also excluded. The surgical procedure was nearly always performed by medical residents at the department privileged to perform the aforementioned surgery. Careful dissection of the surgical layers with proper hemostasis was performed, especially for those patients who had previous pelvic surgeries and particularly multiple previous CS, where some sort of adhesions was expected. There was clear instructions by the department staff forbid-

Table 1. Demographic data of patients

\begin{tabular}{lcc}
\hline Parameter & With drain & Without drain \\
\hline Age (years), mean (range) & $28.3(19-44)$ & $29.1(17-45)$ \\
\hline Gravidity, mean & $7 \pm 3.2$ & $7 \pm 3.3$ \\
\hline Parity, mean & $5 \pm 2.2$ & $5 \pm 1.4$ \\
\hline BMI (kg/m²), mean (range) & $32.2(22-36)$ & $33.00(21-38)$ \\
\hline $\begin{array}{l}\text { Gestational age (weeks), } \\
\text { mean (range) }\end{array}$ & $38.1(29-41)$ & $38.2(28-41)$ \\
\hline Hospital stay (days), mean & 4.8 & 3.1 \\
\hline Operative time (min), mean & 68.6 & 63.8 \\
\hline Hemoglobin level & 10.3 & 10.6 \\
\hline Total & 124 & 121 \\
\hline
\end{tabular}

Table 2. Indication for cesarean section

\begin{tabular}{lcc}
\hline Indication & With drain & Without drain \\
\hline Repeat cesarean & 57 & 54 \\
\hline Failure to progress & 18 & 17 \\
\hline Fetal distress & 17 & 16 \\
\hline Abnormal presentation & 10 & 9 \\
\hline Ante partum hemorrhage & 6 & 7 \\
\hline Patient request & 11 & 12 \\
\hline Others & 5 & 6 \\
\hline Total & 124 & 121 \\
\hline
\end{tabular}

Table 3. Indication favoring drain insertion

\begin{tabular}{lcc}
\hline Feature & Number & Percentage \\
\hline Obesity (BMI > 25) & 15 & 12.1 \\
\hline Difficult homeostasis & 29 & 23.4 \\
\hline Bleeding diathesis & 4 & 3.2 \\
\hline Consultant's preference/no indication & 69 & 55.6 \\
\hline $\begin{array}{l}\text { Previous history of postoperative } \\
\text { intra-abdominal infection }\end{array}$ & 7 & 5.7 \\
\hline Total & 124 & 100 \\
\hline
\end{tabular}

ding the closure if any suspicion of continuous oozing or bleeding exists. Group I with a total number of 124 patients had intra-abdominal drain insertion during the CS, while the group II with a total number of 121 patients had the same procedure performed, but without drain insertion upon strict prohibited instructions by their consultants. When a decision for delivery by CS is approved by a consultant, a written consent for surgery is taken, after a thorough explanation of the indication and the procedure to the patients. Another consent is also taken by the anesthetists, as most of our surgical deliveries usually occur under spinal anesthesia, then the patient would consequently be transferred to the operative theatre. The CS procedure at our hospital is usually performed in a standardized fashion, utilizing the following steps; opening the skin of the anterior abdominal wall, then the subcutaneous layer, the rectus sheath, the visceral peritoneum, then the loose peritoneum over the lower segment picked up by forceps and opened, the vesico-uterine pouch is identified and the bladder pushed downwards and protective metallic retractor used, then a small incision in the lower segment followed by a central curvilinear transverse incision about $10-12 \mathrm{~cm}$ length, artificial rupture of membranes then follows, delivery of the fetus, and lastly removal of the placenta, and closure of the uterine incision in two layers by vicryl 1 sutures. Closure of the visceral peritoneum over the lower segment is not a common practice. Irrigation and hemostasis follows, then insertion of the abdominal drain if applicable, though there are no clear guidelines about this issue and the decision is referred to the operator with special attention to the consultant on call or the consultant in charge of the patient. The drain used is a $10 \mathrm{~mm}$ Jackson-Pratt (JP) drainage tube, a $10 \mathrm{~mm}$ skin incision is made in the lateral lower quadrant with a scalpel, and the drain is placed inside the intraperitoneal space and passed through this separate skin incision. Lastly, closure of the anterior abdominal wall in layers follows.

\section{Results}

A total of 245 patients were eligible to enroll in this study, subdivided in two groups; 124 patients in group I with drain insertion, and 121 patients in group || with no drain insertion. The demographic data of both groups are summarized in Table 1, with almost similar figures of the parameters except for a longer operative time and longer hospital stay for the drained group. In Table 2 , the indications for CS on both groups were clearly reported as well as the type of uterine incision (transverse vs. midline) the nature of the procedure (elective vs. emergency), and factors favoring drain insertion were also reported in Tables 3 and 4 . In the drained group, the drain was removed in 93 (75\%) patients on the first day postoperatively, and was removed after 
5 days in $3(2.5 \%)$ patients (Table 5$)$. The postoperative complications are listed in Table 6 . Among group I, a total number of 10 (8\%) patients reported to have complications related to drain insertion were as follows; 2 patients developed significant bleeding at the site of insertion which necessitated operation and evacuation of the hematomas, 2 patients had drain avulsion at the time of removal which necessitated surgical exploration to remove the plastic piece of the drain, 2 patients had hernia formation which necessitated surgical correction to close the hernia, 2 patients had drain site infection that needed a long course of broad spectrum antibiotics usage, one patient suffered a pelvic abscess that necessitated surgical incision and drainage, and one patient had severe pain at the site of insertion that routinely prescribed postoperatively.

\section{Discussion}

The result of this study were in favor of our view to retract from any dilemmatic intra-abdominal drain insertion during CS. Setting the pros (if any) vs. the cons of such procedure; a very narrow window exist for its use, while its routine use should only be a part of history, for it carries significant complications. Its routine use should not be employed in training facilities for new physicians, but rather for the acquisition of surgical skills. The demographic data showed that its use yielded a longer operative time in group I compared to group II; 68.6 minutes vs. 63.8 minutes respectively. The mean hospital stay was also significantly longer in group II compared to group I; 4.8 days vs. 3.1 days, respectively. The rate of complications was much different; both immediate as significant drain site bleeding that occurred in 2 patients (1.6\%) which necessitated further surgical interference, a percentage higher than that reported by Drukker et al. (0.5\%) [3] and those late occurring including drain avulsion at the time of removal which also necessitated further surgical interference. One case reported marked drain kinking, that interfere with its function, and another case were 3 stitches mistakenly passed through its lumen while trying to fix it the abdomen. Pain necessitating extra doses of analgesia and local infection were reported in 1 case $(0.8 \%)$ and 2 cases $(1.6 \%)$ respectively. This percentage goes in line with previous results by Nora et al. and Gates et al. [4, 5].

Our frightening and life threatening complication of Fallopian tube herniation was reported in 2 subjects of this study (1.6\%) which harmonies with a previous case report published by Saint et al. [6]. Drainage is undoubtedly associated with a huge psychological mal-being and anxiety especially for those who have it placed for longer periods. It is also associated with an economical burden associated with the need for longer hospital stay. An old review conducted by Enkin to evaluate its role in CS as a routine practice that included 2 trials
Table 4. Type of incision/type of cesarean

\begin{tabular}{lcc}
\hline Type & With drain & Without drain \\
\hline Low transverse & 119 & 117 \\
\hline Mid-line & 5 & 4 \\
\hline Total & 124 & 121 \\
\hline Elective & 88 & 91 \\
\hline Emergency & 36 & 30 \\
\hline Total & 124 & 121 \\
\hline
\end{tabular}

Table 5. Postoperative date removal of the drain

\begin{tabular}{lcc}
\hline Day & Number & Percentage \\
\hline First day & 93 & 75 \\
\hline Second day & 21 & 16.9 \\
\hline 2-3 days & 7 & 5.6 \\
\hline 4-5 days & 3 & 2.5 \\
\hline Total & 124 & 100 \\
\hline
\end{tabular}

Table 6. Postoperative drain insertion complications

\begin{tabular}{lcc}
\hline Complication & Number & Percentage \\
\hline Bleeding & 2 & 20 \\
\hline Pelvic abscess & 1 & 10 \\
\hline Avulsion & 2 & 20 \\
\hline Organ herniation & 2 & 20 \\
\hline Severe site pain & 1 & 10 \\
\hline Drain site infection & 2 & 20 \\
\hline Total & 10 & 10 \\
\hline
\end{tabular}

concluded that its use may be of benefit if homeostasis is in doubt, but routine use was shown to have no role [7]. A retrospective study by Drukker et al. on the other hand showed that its use must be employed in difficult surgeries and that it is associated with a shorter interval to relaparotomy [3]. Our group II study variants' results however conflicted with any routine/prophylactic insertion and showed no surgical complications, and made us keen to state that no drain insertion greatly out weights its ignorant employment. Our results go in harmony with Gates and Anderson findings that suggested that the routine use of wound drains at CS does not confer any substantial benefit to the women involved [5].

The placement of the abdominal drainage tube after CS should not be a routine precautionary procedure for the possibility of a surgical complication. It should not be within the policy of training programs for new physicians, but rather the promotion and acquisition of surgical skills. Among the demographic data, there was longer operating time in the group I compared to group II; 68.6 minutes vs. 63.8 minutes respectively. Also, the mean hospital stay was significantly differ between both groups as it was longer in group I in comparison to group II; 4.8 days vs. 3.1 days, respectively. The surgi- 
cal complications of our study clearly show the negative effect of the placement of the abdominal drains as we noticed the presence of surgical complications in the first group connected to drain insertion; part of them as immediate complications like what happened for $2(1.6 \%)$ patients who developed significant drain site bleeding mostly at the time of insertion that needed surgical interference, with the same incidence of avulsion of a part of the drain at the time of removal that necessitated surgical intervention, as three sutures passed through the lumen of the drain in one case and marked kinking of the drain was noted in the other one. One (0.8\%) patient developed severe site pain and 2 patients had local infection, with similar observations in the study conducted by Nora et al. [4], while late complications involved fallopian tube herniation at the site of drain insertion in $2(1.6 \%)$ patients, with the same content of the case report published by Pradeep et al. [6] and abscess formation in the pelvis due to infection from the site of the tube. It is not excluded for possible doubts the psychological effects of the existence of the tube discharge abdominally especially for patients who kept the tube for several days, and increased the value of the therapeutic bill as a result of increased duration of stay. An old Cochrane systemic review conducted by Enkin, to evaluate role of routine drainage in CS in which 2 trial included $[7,8]$ Enkin concluded that the use of such drainage may be of benefit if hemostasis is inadequate, but a benefit from routine use has not been established [9]. We think it's clear that no drainage at all is better than the ignorant employment of it. This is a clear introduction to respect the results of the second group as we did not notice any surgical complications in the absence of drain insertion.

Thus, the rationale behind abdominal drainage following CS has been the value afforded by drains in forewarning the surgeon of potential intra-abdominal complications [10]. Traditionally, surgeons have resorted to placing multiple drains. However, as in our current study, drains have been implicated in the causation of local pain, ascending infection $[4,11]$ and interference with patient ambulation [12]. With the advances that are being made in many areas of medicine, the surgeon must be familiar with infectious diseases of the peritoneal cavity, which have increased in scope and complexity [13].

A potential limitation of our study is the difficulty in finding relevant information due to scarcity in research in this topic. Even when drainage was studied, post discharge surveillance was not employed. Moreover, most of the drainage systems employed were preventive and relied on previous personal experiences of complications; hence their insertion was driven by fear and poor surgical techniques rather than conveying any actual benefits, especially with the employment of new rules in medical litigation. Furthermore, prophylactic drains have been placed as a warning sign to detect post- operative bleeding which may be a reaction to a previous case or an old experience due to the possibility of medical accountability without an evidence based background. Lastly, we declare that confounding factors such as the presence of medical comorbidities/ obesity attribute to the presence of complications associated with drain insertion and could be a source of bias.

Thus, we concluded that intra-abdominal drains should not serve on behalf of the surgeons eyes as a forewarning of intra-abdominal complications. The need to drain has always been a controversial subject in the field of surgery. There are those who believed all peritoneal operations should be drained, those who felt drainage is useless, and those who sit on force and insert a drain as a safety valve or perhaps as a sop to their onsciera. Their discussions are largely based on personal opinions. History furnished with a picture that demonstrates the problems which our forefather faced and which face us today.

\section{Conclusions}

Intra-abdominal drain insertion after CS is a rather unjustified diligence, as the evidence is present that abdominal drains are potentially harmful and their use should be remotely restricted to only when a clear indication is present, which is the exception rather than a rule. Drains are not substitutes for careful hemostasis and meticulous dissection. Good surgical techniques with adequate hemostasis, the elimination of dead space, and the use of prophylactic antibiotics obviates the need for drains in most patients. Application of good surgical skills especially in cases of patients with multiple previous CS with adhesions must overcome the need for the application of drains. Proper inspection of the surgical field, site of dissected adhesions, the nearby bladder and bowel to recognize any possible injury would be markedly superior to the habitual drain insertion. When drainage is employed, it should be of the most efficient, closed type, and the drain must be removed at the earliest, safest time after operation. The placement of the abdominal drainage tube to diagnose internal bleeding or peritonitis is a modest surgical skill and should be discontinued.

\section{Acknowledgments}

The authors would like to thank all the patients for their willingness to participate in the study and their patience.

The study was supported by Jordan University Hospital (in-kind).

\section{Disclosure}

The authors report no conflict of interest. 


\section{References}

1. O'Connor TW, Hugh TB. Abdominal drainage: a clinical review. ANZ J Surg 1979; 49: 253-260.

2. Schein M. To drain or not to drain? The role of drainage in the contaminated and infected abdomen: an International and personal perspective. World J Surg 2008; 32: 312-321.

3. Drukker L, Shen O, Rottenstreich M, et al. To drain or not to drain: intraperitoneal closed-suction drainage placement during cesarean delivery. J Matern Fetal Neonatal Med 2019; DOI: 10.1080/14767058.2019.16775 91.

4. Nora PF, Vanecko RM, Bransfield JJ. Prophylactic abdominal drains. Arch Surg 1972; 105: 173-176.

5. Gates S, Anderson ER. Wound drainage for caesarean section. Cochrane Database Syst Rev 2013; 2013: CD004549.

6. Saint P, Faridi MS, Agarwal N, et al. Drain site evisceration of fallopian tube, another reason to discourage abdominal drain: report of a case and brief review of the literature. Tropical Doctor 2012; 42: 122-124.

7. Enkin MW. Closed suction wound drainage at caesarean section. Pregnancy and Childbirth Module. Cochrane Database Syst Rev 1995; Disk Issue 2.

8. Loong RL, Rogers MS, Chang AM. A controlled trial on wound drainage in Caesarean section. Aus New Zeal J Obstet Gynecol 1988; 28: 266-269.

9. Saunders NJ, Barclay C. CLOSED suction wound drainage and lower segment caesarean section. Br J Obstet Gynecol 1988; 95: 1060-1062.

10. Bindal J, Munda G. A clinical study to compare drain versus no drain in post cesarean section. Int J Reprod Contracept Obstet Gynecol 2017; 6: 3903-3906.

11. Raves JJ, Slifkin M, Diamond DL. A bacteriologic study comparing closed suction and simple conduit drainage. Am J Surg 1984; 148: 618-620.

12. Buchler MW, Friess $\mathrm{H}$. Evidence forward, drainage on retreat: Still we ignore and drain!? Ann Surg 2006; 244: 8-9.

13. Sharma R, Gukeria K, Suneja A, Bhartiva V. Post-Caesarean Drain Placement - Minor Procedure Leading to Major Complication. J Clin Diagn Res 2017; 11: QD03-QD04. 